\title{
Chitosan temperature-sensitive gels: reduce the burst release of microspheres containing lornoxicam and enhance drug targeting
}

\begin{abstract}
The aim of this study was to prepare fine intra-articular administration chitosan/ $\beta$ glycerophosphate-temperature-sensitive gelatine combined with PLGA microspheres containing Lornoxicam and to evaluate the possibility of those gelatine as drug delivery for reducing the burst release of the microspheres in target site and improving the retention drug concentration. The prepared microspheres as per previous study has a certain burst release and the temperature-sensitive gelatine has good release effect, so combining system we prepared this study were evaluated in terms of appearance characteristics, in vitro drug release, in vivo joint cavity leakage and drug retention. The optimal prescription containing microspheres exhibited sol-semi-solid transition at $37^{\circ} \mathrm{C}$ and quickly turn into gel within $5 \mathrm{~min}$, which could reduce the initial burst at the beginning of intra-articular injection and delay drug release during the treatment with rats. The drug retention and joint cavity leakage outcomes in rats reveal that the combined delivery system may be used as a potential drug delivery system for treat osteoarthritis.
\end{abstract}

Keywords: burst release, intra-articular administration, temperature-sensitive gelatine, lornoxicam loaded PLGA microspheres
Volume 5 Issue I - 2018

\author{
Shengjun Zhu, ${ }^{1,2}$ Hong Tian, ${ }^{1,2}$ Mingjin Dou, ${ }^{2}$ \\ Guihua Huang ${ }^{3}$ \\ 'School of Medicine and Life Sciences, University of Jinan- \\ Shandong Academy of Medical Sciences, China \\ ${ }^{2}$ Affiliated Hospital of Shandong Academy of Medical Sciences, \\ China \\ ${ }^{3}$ School of Pharmaceutical Sciences, Shandong University, China
}
Correspondence: Mingjin Dou,Affiliated Hospital of Shandong Academy of Medical Sciences, China, No.38 Wuyingshan Road, Ji'nan, Shandong Province, China, Email mingjindou1965@126.com

Co-correspondence: Guihua Huang, School of Pharmaceutical Sciences, Shandong University, China, No.44Wenhua Xi Road, linan, Shandong Province, China, Email hgh2003@I26.com

\section{Introduction}

Lornoxicam (Lnxc), a novel type of non-steroidal antiinflammatory drug developed by comely, was first listed in Denmark in October 1997 (trade name xefo). Clinically, it is used to treat rheumatoid, rheumatoid arthritis and osteoarthritis. The Lnxc with low drug concentration has a short half-life ${ }^{1}$ after oral administration, leading to much side effect and therapy activity tended to be worse, therefore, the method of local injection of intra-articular cavity was concerned and widely used. ${ }^{2}$ Intra-articular local administration of Lnxc is expected to be superior to systemic delivery in pain relieving and less adverse effects. ${ }^{3-5}$ Intra-articular drug delivery system may be a better therapeutic approach for the treatment of a variety of knee pain. Through the animal study we can conclude that there were no side effects of intra-articular administration of Lnxc. ${ }^{6}$ However, intraarticular injection is a kind of local treatment method, which can inject the drugs into the joint cavity of the patients directly. It is difficult to achieve continuous therapeutic effect after intra-articular injection, because a fast and almost complete absorption of Lornoxicam from the joints into the systemic circulation, so it often needs to be repeated several times.

Poly (lactide-co-glycolide acid) (PLGA) microspheres are efficient delivery systems for controlled release of low molecular weight drugs, ${ }^{8}$ and its drug release considers both drug dissolution and the diffusion of dissolved active principle through the polymeric matrix. PLGA microspheres have been proved to have good therapeutic effect in the treatment of osteoarthritis. ${ }^{10-12}$ For instance, the SFN-PLGA microspheres system devised in this study that can be used for treating osteoarthritis, which indicates that SFN-PLGA microspheres delayed the progression of surgically-induced osteoarthritis in rats. ${ }^{13}$ The intraarticular delivery of Lnxc loaded chitosan microspheres can minimize associated side-effect after prolonged oral administration. Compared to Lornoxicam solution, chitosan microspheres of Lnxc showed longterm anti-inflammatory effect after injected intra-articular, with the advantages of alleviating histological, inflammatory and biochemical parameters of osteoarthritis. ${ }^{14}$

Relevant studies showed that the amount of drugs inside the microspheres sharply decreased with time. ${ }^{15}$ If there is a sudden drug release in the body after injection, leading to the initial drug concentration increased suddenly in the blood, which would result in a various of adverse reactions or even reach the poisoning drug concentration. Study on pharmacokinetics results of this experiment have shown that the much of drug leakage to plasma at $0.5 \mathrm{~h}$ after intra-articular injection of Lornoxicam loaded PLGA microspheres (Lnxc-PLGA-MS), which demonstrates that a certain burst release in vivo. Moderate sudden release of microspheres could improve the therapeutic effect; however, excessive drugs would lead to side-effect when leaks to the systemic circulation. Local injection of temperaturesensitive hydro-gels containing drugg ${ }^{16,17}$ or other materials has been widely studied as a sustained controlled release formulations of intra-articular injection, and has a preferable prospect of application. Intra-articular administrated chitosan temperature-sensitive hydrogels combined with alginate microspheres and hydrogels as a drug delivery system for promoting the anti-inflammation effect. Compared to solution group, drugs loaded hydro-gels released delayed $5 \mathrm{~d}$. The potential drug delivery system for improving the therapeutic effect of diclofenac sodium and suggested an important technology platform for intra-articular administration. ${ }^{18}$

Our group has developed a sustained release microspheres for Lnxc, which was intra-articular delivery system with the goal of localizing Lnxc in joint cavity over a few days. In our previous study, Lnxc loaded PLGA microspheres were developed, characterized and joint cavity leakage in rabbits and drug retention in the synovial fluid in rats 
were evaluated, which verified the controlled effect in local site by reducing the leakage of drugs from joint cavity to blood circulation. ${ }^{19}$ Lnxc loaded PLGA microspheres or blank microspheres were injected into the articular cavity, there was no accumulation of filtration liquid, no proliferation of blood vessel and fiber were detected within 30 $\mathrm{d}$, which showed that they had good biocompatibility. At the same time, Lnxc PLGA microspheres can also repair the articular cartilage damaged by papain. ${ }^{20}$ Furthermore, chitosan/ $\beta$-glycerophosphate $(\mathrm{CS} / \beta$-GP)-temperature-sensitive gelatine were preparation successfully, and has the characteristics of physiological neutrality. The gels underwent a gelation to form semisolid state at a specific site after administrated into the body, responded to temperature at $37^{\circ} \mathrm{C}$ within $5 \mathrm{~min}$. The $(\mathrm{CS} / \beta$-GP)-temperature-sensitive gelatine was used as a drug carrier for intra-articular injection, with ideal gelling temperature and better sustained release effect. ${ }^{21}$ On the basic of our previous work, in this study, the aim was to study the treatment of osteoarthritis with (CS/ $\beta$-GP)-temperature-sensitive, gelatine loaded Lnxc-PLGA-MS (Lnxc-PLGA-MS-gels). Compared to Lnxc-PLGAMS, Lnxc-PLGA-MS-gels could reduce the initial burst effectively within $8 \mathrm{~h}$, and drug concentration was higher than it in targeted site on the overall trend, which demonstrates that the delivery system of temperature-sensitive gels loaded Lnxc-PLGA-MS can achieve better therapeutic effect. The drug delivery system could reduce the burst release, and achieve a better sustained release effect.

\section{Materials and methods}

\section{Materials}

Lnxc was purchased from Hubei Yuanchengsaichuang Technology co. Ltd Hubei, China. Poly (D,L-lactic-co-glycolic) (PLGA 75:25 Av, MW:15,000, $25 \mathrm{kDa}$ ) was purchased from Advanced Polymer Materials Inc. Polyvinyl alcohol (PVA, MW: 13,000 23,000, $87 \% \sim 89 \%$ part of partial alcoholysis type ) was provided by Sigma Chemical Co. Ltd. Chitosan was purchased from Sigma-aldrichl Inc, China. $\beta$-Glycerophosphate was purchased from Shanghai Hengfei Biological Technology co. Ltd, Shanghai, China. Sodium azide was purchased from Shanghai Shanjing Molecular biotechnology co, Ltd. Tween- 80 was purchased from Laiyang Economic development zone factory, China. The other chemicals were of analytical reagent grade or higher.

Animal: Female Wistar rats $(200 \pm 20 \mathrm{~g}$, were provided by the Medical Animals Test Center of Shandong University) were used for the in vivo pharmacokinetics and drug retention studies. The animal experiment protocol was reviewed and approved by the Institutional Animal Care and Use Committee of Shandong University.

\section{Preparation}

Preparation of dried PLGA microspheres loaded with Lnxc: Lnxc-PLGA-MS were prepared by a process involving solid-in-oil-in-water $(\mathrm{S} / \mathrm{O} / \mathrm{W})$ multiemulsion as per the previous research results of our research group. ${ }^{19,20} \mathrm{~A}$ sample of $400 \mu \mathrm{l}$ water including $4 \mathrm{mg}$ Lnxc was suspended in $2 \mathrm{ml}$ of $4 \%$ PLGA (W/V) solution dissolved in dichloromethane. After stirring at high speed $(10,000 \mathrm{rpm})$ for $1 \mathrm{~min}$, the resulting primary emulsion was injected to $10 \mathrm{ml} 1 \%$ PVA solution to form emulsion, meanwhile, stirring at high speed $(5,000 \mathrm{rpm})$ for $1.5 \mathrm{~min}$. The multi emulsion was continuously stirred for $3 \mathrm{~h}$ at room temperature $\left(25^{\circ} \mathrm{C}\right)$ under ambient pressure until all the dichloromethane had evaporated. The solidified microspheres were recovered by centrifugation and washed three times with distilled water. Finally, the microspheres were dispersed evenly with appropriate amount of distilled water, using $2 \%$ mannitol as a protective agent, then the dried microspheres were prepared by freeze drying method (pre-freezing $12 \mathrm{~h}$ under $-80^{\circ} \mathrm{C}$, then placed in the freeze dryer under $-50^{\circ} \mathrm{C}$, freeze-drying for 24 hours), and dried in a freeze-drier.

Preparation of temperature-sensitive gels containing Lnxc-PLGA-MS: Temperature-sensitive gels were successfully prepared as per the previous research results of our research group. ${ }^{19}$ The proper amount of chitosan was placed in acetic acid solution of $1 \mathrm{ml} 0.1 \%$ $\mathrm{mol} / \mathrm{L}$, which is fully dissolved under the action of magnetic stirring. In an ice bath $\left(10^{\circ} \mathrm{C}\right)$, the amount of $\beta$-GP solution was slowly dropped into chitosan acetate solution, and the blank CS/ $/$-GP temperature-sensitive gels were prepared after stirring for $30 \mathrm{~min}$, then the prepared blank temperature-sensitive gels were stored at $4^{\circ} \mathrm{C}$. The temperature-sensitive gels containing Lnxc-PLGA-MS was prepared by mixing the Lnxc-PLGA-MS and blank temperature sensitive gels.

\section{Properties and characteristics}

Thermal Pproperties of microspheres: DSC (CDR-4P) was performed on free Lnxc, mixture of Lnxc and accessories, blank microspheres and Lnxc-PLGA-MS to determine the thermal behavior. Using the differential thermal analyzer to take $\mathrm{Al}_{2} \mathrm{O}_{3}$ as reference, sample A: (Lnxc); Sample B: (mixture of lnxc and excipients); Sample C: (accessories); Sample D: (Lnxc-PLGA-MS) 6mg, respectively. Samples were heated from $40^{\circ} \mathrm{C}$ to $400^{\circ} \mathrm{C}$ at $10^{\circ} \mathrm{C} / \mathrm{min}$, to verify the formation of Lnxc-PLGA-MS.

Appearance shape and particle size analysis of microspheres: Taking proper amount of microspheres into the penicillin bottle, then observe the state of the microspheres before and after freeze-dried, including fresh-prepared Lnxc-PLGA-MS, the Lnxc-PLGA-MS freeze-dried power and the suspension of Lnxc-PLGA-MS powder (dispersed with distilled water), respectively. Taking proper amount of fresh-prepared Lnxc-PLGA-MS and freeze-dried powder, respectively. Put them on the glass slide, and add a drop of deionized waterand tar to disperse the microspheres, then observe the morphology of the microspheres under the optical microscope. In addition, the morphology of Lnxc-PLGA-MS was characterized by scanning electron microscope. Particle size distributions were determined by laser light scattering (Mastersizer 2000). The proper amount of PLGA microspheres were dispersed in distilled water. All size distributions were presented in the volume-weighted mode and the particle size distribution.

\section{Release of drugs in vitro and in vivo}

In vitro drug release: The drug release of Lnxc-PLGA-MS and chitosan temperature-sensitive gels containing Lnxc-PLGA-MS was studied by dynamic membrane dialysis. ${ }^{22,23}$ The Lnxc-Sol, Lnxc-PLGA-MS and Lnxc-PLGA-MS-gels were kept in a dialysis membrane (MD34, 8,000-14,000), which concentrations were $1 \mathrm{mg} / \mathrm{ml}$ and all the volume is $2 \mathrm{ml}$. The dialysis bag containing Lnxc-PLGA-MS-gels was placed in constant temperature water bath at $37^{\circ} \mathrm{C}$ for $5 \mathrm{~min}$ to make the drug temperature sensitive gels to the state of gels. Then, all systems were immersed in $28 \mathrm{ml}$ of $\mathrm{pH} 7.4$ buffer solution, respectively. All the vials kept at $37^{\circ} \mathrm{C}$ under continuous magnetic stirring of $100 \mathrm{rpm}$. At a regular interval of time, $5 \mathrm{ml}$ of receiver solution was withdrawing and same volume of fresh medium was placed. The concentration of Lnxc in the release medium was measured by UV-Visible spectrophotometer at $387 \mathrm{~nm}$. The mean calculated values were obtained from 3 replicates. In order to determine the release mechanism 
of Lnxc from PLGA microspheres and the Lnxc-PLGA-MS-gels, the release data were analyzed by model-dependent methods.

Determination of targeting efficiency: Female Wistar rats $(200 \pm 20 \mathrm{~g}$, were provided by the Medical Animal Test Center of Shandong University) were used for the joint cavity leakage and drug retention studies in the joint cavity. The animal experiment protocol was reviewed and approved by the Institutional Animal Care and Use Committee of Shandong University. Eighty-Seven Wistar rats were randomly divided into three groups and every group has three rats as the same level:

a. Lnxc solution groups.

b. Lnxc-PLGA-MS suspension groups.

c. Lnxc-PLGA-MS-gels groups. All the concentration of Lnxc was $5 \mathrm{mg} / \mathrm{ml}$, the dosage was $4 \mathrm{mg} / \mathrm{kg}$.

The rats were anaesthetized with ether, three different preparations of $0.2 \mathrm{ml}$ of left articular cavity were injected in each group. After the administration, blood was taken from the jugular vein sinus at $0.5,2$, $4,6,8,12,24,48,96,146$ and $336 \mathrm{~h}$. For each preparation and each sampling time point, three rats were treated with a single dose of $4 \mathrm{mg} /$ $\mathrm{kg}$ after intra-articular injection administration of Lnxc-Sol, LnxcPLGA-MS and Lnxc-PLGA-MS-gels. $0.2 \mathrm{ml}$ acetonitrile and $0.1 \mathrm{ml}$ methanol-sodium acetate $(3: 2)$ were added to an aliquot $(0.2 \mathrm{ml})$ of each plasma sample and mixed for $3 \mathrm{~min}$ by vortex to extract Lnxc. Following centrifugal at $12,000 \mathrm{rpm}$ for $10 \mathrm{~min}$, the supernatant was filtered by $0.22 \mu \mathrm{m}$ organic filter, then $20 \mu 1$ solution was injected into the HPLC column and the content of Lnxc in plasma was measured. The treatment of joint tissue and synovial fluid is same as above. The concentration of Lnxc was measured by the method of reversedphase HPLC with the column, InertSustain ${ }^{\circledR}-\mathrm{C} 18$ column $(4.6 \mathrm{~mm} \times$ $250 \mathrm{~mm}$ ). Lnxc was monitored wavelength of $376 \mathrm{~nm}$. The mobile phase was composed of methanol and $0.05 \mathrm{~mol} / \mathrm{L}$ Sodium acetate $(60: 40, \mathrm{v} / \mathrm{v})$, the flow rate was $1.0 \mathrm{ml} / \mathrm{min}$. The number of theoretical plates is not less than 4,000. All chromatography was performed at room temperature.

\section{Results and discussions}

\section{Preparation}

Preparation of dried PLGA microspheres loaded with Lnxc: Encapsulation efficiency was defied as the Lnxc found in the microspheres as a percentage of input one. The repeatability test showed good results. An average entrapment efficiency of $(81.53 \pm 1.10) \%$ and an average drug loading of $(5.05 \pm 0.10) \%$ were achieved in the Lnxc-PLGA-MS.

Preparation of temperature-sensitive gels combined with Lnxc-PLGA-MS: This paper prepared temperature-sensitive gels containing Lnxc-PLGA-MS by CS/ $\beta$-GP temperature-sensitive materials, to optimize the prescription and process by evaluation index of gelatine temperature, gelatine time and the nature of needle. The best prescription of the temperature-sensitive gels containing Lnxc-PLGA-MS was $\mathrm{pH} 7.2,3 \% \mathrm{CS}, 60 \% \beta$-GP. Considering the gels would too sticky to injection, $20 \mathrm{mg} / \mathrm{ml}$ of Lnxc-PLGA-MS was chosen to be the final formation. It was found that gels containing microspheres were neutral solutions at room temperature and could quickly turn into semi-solid gels state within $5 \mathrm{~min}$ at $37^{\circ} \mathrm{C}$, the states we could see it clearly in Figure 1. Meanwhile, the characteristics of gels were stabilization and suitable for joint injection, which might be a probable consequence of increased viscosity and intensity of the complex formation caused by the interaction between Lnxc-PLGA-MS, CS and $\beta$-GP.

\section{Properties and characteristics}

Thermal Properties of Microspheres: The change of sample temperature is mainly caused by heat absorption or heat effect in DSC test. In general, phase transition, dehydrogenation reduction and some decomposition reactions produce endothermic effects; the crystallization, oxidation and some decomposition reactions produce exothermic effect. The Figure 2 shows the following results. Thermogram of Lnxc showed a sharp endothermic peak at $222.3^{\circ} \mathrm{C}$, and an obvious endothermic peak of excipients at $315^{\circ} \mathrm{C}(\mathrm{A}$ and $\mathrm{B})$. While there was no peak corresponding to Lnxc in the DSC curve of Lnxc-PLGA-MS (C and D), which shows that the drug are amorphous molecular existed in the microspheres rather than adsorbed on the surface of the microspheres.

Appearance shape and Particle size analysis of microspheres: The photographs of fresh-prepared Lnxc-PLGA-MS.

a. The freeze-dried powder of Lnxc-PLGA-MS.

b. The suspension of Lnxc-PLGA-MS powder.

c. Dispersed with distilled water are shown in Figure 3.
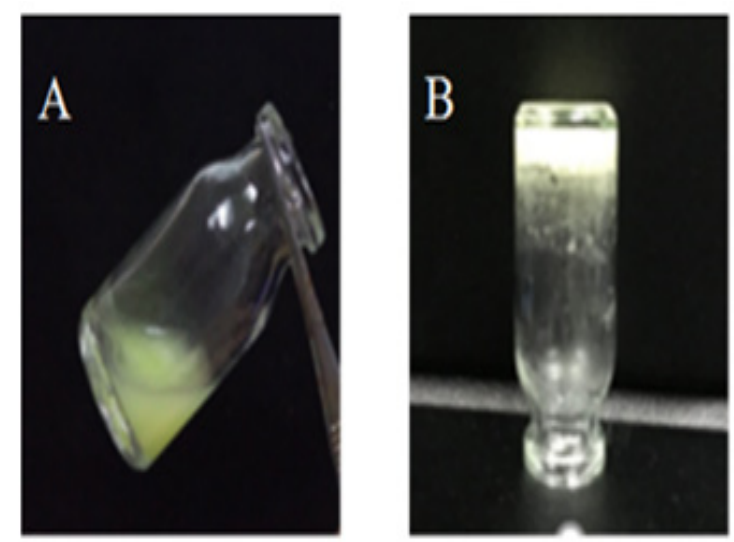

Figure I The appearance of Lnxc-PLGA-MS loaded gels at different temperature $\left(\mathrm{A}: 25^{\circ} \mathrm{C} ; \mathrm{B}: 37^{\circ} \mathrm{C}\right)$.

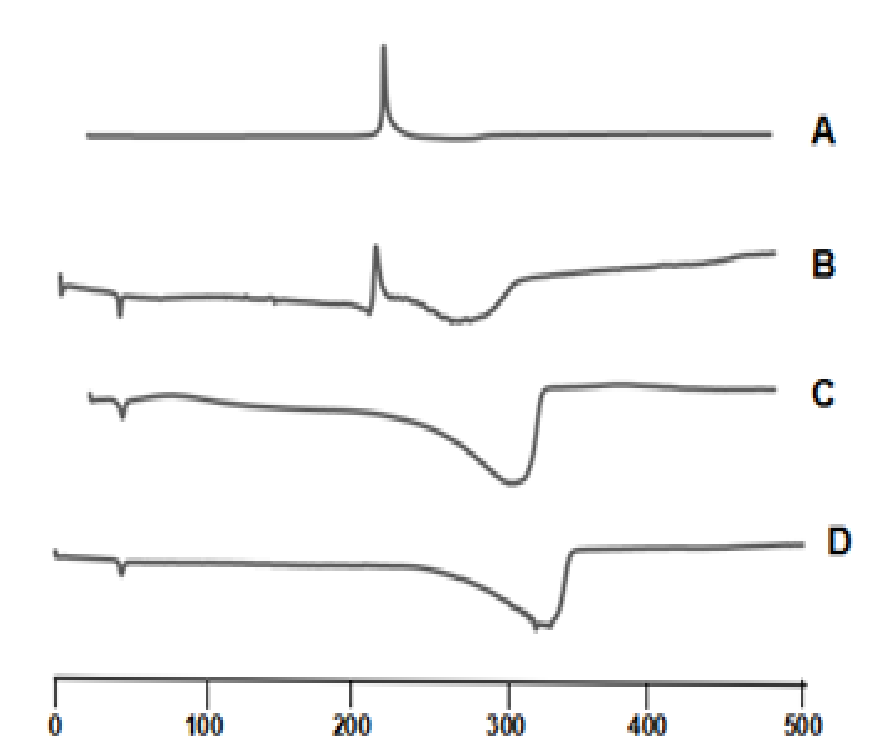

Figure 2 A: The DSC results of Lnxc; B: Mixture of Inxc and excipients; $C$ : Accessories; D: Lnxc-PLGA-MS. 
The photograph of A was suspension uniformly. The photographs of B were loosely yellow powder, displayed good dispensability with distilled water. The morphology of Lnxc-PLGA-MS was observed by optical microscopy, the observation results were displayed in Figure 4 and Figure 5. The image shows that most microspheres have smooth surface of spherical particles with approximate size and uniform dispersion, besides, there is no adhesion among the microspheres. The particle size distribution of Lnxc-PLGA-MS was determined by laser particle size analyzer. Figure 6 displayed the particle size distribution pattern typically for two kinds. Table 1 has shown that the mean particle size of the microspheres was $6.65 \mu \mathrm{m}$ and $6.88 \mu \mathrm{m}$, which was fresh-prepared and freeze-dried microspheres, respectively. The particle size was in normal distribution of both kinds of microspheres. The average particle size and particle size distribution of Lnxc-PLGAMS before and after freeze-dried showed little difference, indicating that the effect of freeze-dried on the particle size of microspheres was small, and did not appear adhesive bond among the microspheres.
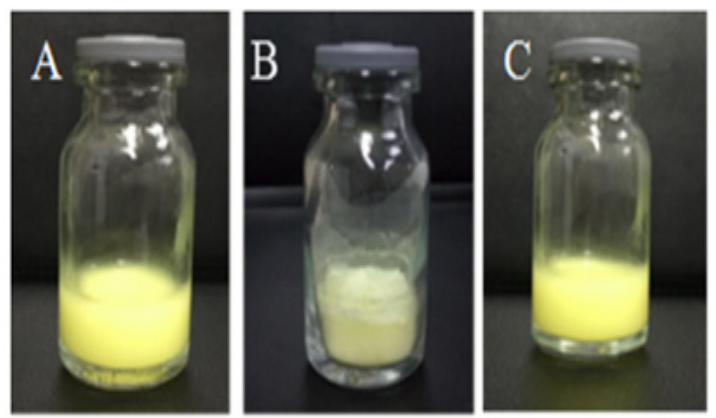

Figure 3 A: Photographs of Lnxc-PLGA-MS: Fresh-prepared; B: Freeze-dried powder; C:The suspension of freeze-dried powder
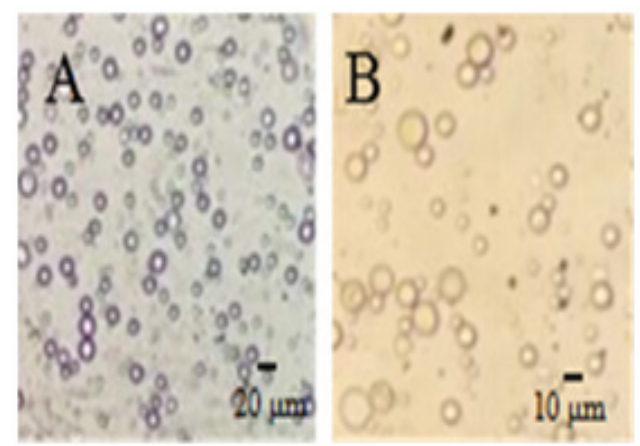

Figure 4 Optical microscopy photograph of fresh-prepared Lnxc-PLGA-MS. A: Deionized water; B: Cedar oil

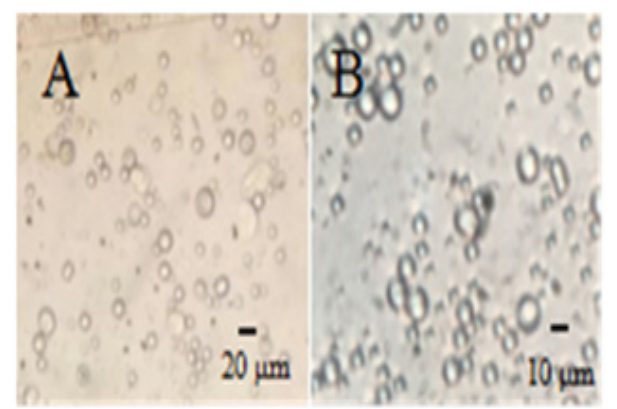

Figure 5 Optical microscopy photograph of freeze-dried Lnxc-PLGA-MS: A: Deionized water; B: Cedar oil
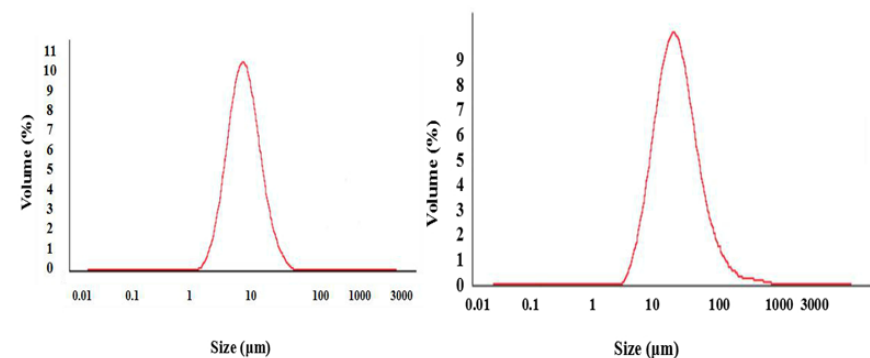

Figure 6 Size distribution of A: Lnxc-PLGA-MS Fresh-prepared microspheres; B: Freeze-dried microspheres.

Table I Mean size of Lnxc-PLGA-MS

\begin{tabular}{lllll}
\hline Sample & I & $\mathbf{2}$ & $\mathbf{3}$ & $\begin{array}{l}\text { Average } \\
\mathbf{\pm S D}\end{array}$ \\
\hline Fresh-Prepared Microspheres $(\mu \mathrm{m})$ & 6.63 & 6.59 & 6.74 & $6.65 \pm 0.06$ \\
Freeze-Dried Microspheres $(\mu \mathrm{m})$ & 6.84 & 6.92 & 6.87 & $6.88 \pm 0.03$ \\
\hline
\end{tabular}

\section{Release of drugs in vitro and in vivo}

In vitro drug release: To determine in vitro drug release of Lnxc from Lnxc-PLGA-MS with or without temperature-sensitive hydro-gels, the cumulative release (\%) of Lnxc was measured at certain time. The release of Lnxc from Lnxc-Sol, Lnxc-PLGA-MS and Lnxc-PLGA-MS-gels were in accordance with the Weibull equation, the regression equation is shown in Table 2 . The equation can be applied to forecast release amount of Lnxc at different time, or calculate the time to release a certain amount of Lnxc, which could predict the status in practical applications. Figure 7 shows the cumulative release of Lnxc from the Lnxc-Sol has a quick release, which was released $(81.40 \pm 0.66) \%$ within $8 \mathrm{~h}$. The results show that the cumulative release of Lnxc from Lnxc-PLGA-MS was $(30.29 \pm 0.66) \%$ in $1 \mathrm{~h}$, and there is a certain burst release. The reason may be that some drugs are spread to the aqueous phase with the solvent during the volatilization of organic solvents, then the free drugs is adhered to the surface of the microspheres or dispersed between the matrix of PLGA microspheres, and the rapid diffusion of the free drugs in the early release of the drug that showing a sudden release. However, the cumulative release of Lnxc from Lnxc-PLGA-MS-gels was $(20.40 \pm 0.35) \%$ in $1 \mathrm{~h}$, and the reason of this slow release action is that the temperature-sensitive gels formed a skeleton structure with gaps after forming a semi-solid gel. The drug needs to go through two steps before entering the release medium, including drug is released from the microspheres slowly and then go through the interstitial skeleton of the gels, and the gels has a certain viscosity, all of which were delayed the release of the drug. In last stage, the release rate of the drugs from the microspheres loaded gels group and the microspheres group tend to be consistent, as the skeleton of the temperature-sensitive gels is eroded constantly, then its effect of delaying release is decreased gradually.

Joint cavity leakage in rats after intra-articular injection administration: The plasma drug concentration of the solution group in 0.5-96 $\mathrm{h}$ was significantly higher than that in the microspheres group and the Lnxc-PLGA-MS-gels group. Especially in the first 8h, the concentration of Lnxc in the plasma of the microspheres group was higher than that Lnxc-PLGA-MS-gels, the concentration differences of those was larger. Meanwhile, the sudden release effect of the microspheres would increase the drug concentration suddenly in the plasma, resulting in the blood concentration of microspheres group 
was higher than that in the Lnxc-PLGA-MS-gels group at the former $8 \mathrm{~h}$. A linear relationship between Lnxc concentration in plasma and ratios of peak areas of Lnxc over the internal standard was obtained in the concentration range of $60-3,000 \mathrm{ng} / \mathrm{ml}(0.999)$ by the HPLC assay. The plasma samples of different time points were measured, using a plasma standard solution of $750 \mathrm{ng} / \mathrm{ml}$ as the control, the concentration of Lnxc in plasma at various time points was calculated, the results are shown in Figure 8. After the targeted injection of the joint cavity, the smaller the drug concentration that enters the blood circulation, the more the amount of drugs retard in the joint cavity. The drug is absorbed into the systematic circulation from the joint cavity rapidly after the joint cavity injection of Lnxc solution, so the drug concentration in the plasma is relatively high, comparing with Lnxc-PLGA-MS groups and Lnxc-PLGA-MS-gels groups both have lower plasma concentration. Figure 8 shows that the Lnxc-PLGA-MS-gels significantly reduces the leakage of drugs to the blood, increases the drug concentration of the joint cavity, and significantly reduces the sudden release effect of Lnxc-PLGA-MS.

Drug retention in the articular tissues and synovial of rats after intra-articular injection administration: To evaluate whether Lnxc-PLGA-MS-gels were release drugs more slowly, and reduce the sudden burst of the Lnxc-PLGA-MS in the joint, articular tissues and synovial of rats were collected and handled for further analysis. From Figure 9 and Figure 10, we could seen that there were significant differences between three groups. Lnxc-Sol groups and Lnxc-PLGA-MS groups were decreased faster than the Lnxc-PLGA-MS-gels groups, and the Lnxc-PLGA-MS-gels has relatively higher drug concentration than the other two groups constantly. As shown in Figure 9 and Figure

Table 2 The regression equation of Lnxc-Sol/Lnxc-PLGA-MS/Lnxc-PLGA-MS-gels release in vitro

\begin{tabular}{|c|c|c|c|}
\hline & Model & Equation & $\mathbf{R}$ \\
\hline \multirow[t]{5}{*}{ Lnxc-PLGA-MS } & Zero-Order Kinetics & $\mathrm{Q}=0.21 \mathrm{t}+28.995$ & 0.897 \\
\hline & First-Order Kinetics & $\ln (100-Q)=-0.005 \mid t+4.27$ & 0.965 \\
\hline & Higuchi Equation & $\mathrm{Q}=4.06 \mathrm{t}^{1 / 2}+\mid 8.23$ & 0.974 \\
\hline & Weibull Equation & $\ln \ln (1 /(I-Q / 100)=0.40 \ln t-1.78$ & 0.994 \\
\hline & Pep Ritger-pas Equation & $\ln Q=0.30 \ln t+2.80$ & 0.988 \\
\hline \multirow[t]{5}{*}{ Lnxc-Sol } & Zero-Order Kinetics & $Q=4.06 t+47.09$ & 0.78 \\
\hline & First-Order kKinetics & $\ln (100-Q)=-0.12 t+3.95$ & 0.94 \\
\hline & Higuchi Equation & $Q=19.38 t^{1 / 2}+27.86$ & 0.948 \\
\hline & Weibull Equation & $\ln \ln (I /(I-Q / 100)=0.55 \ln t-0.56$ & 0.997 \\
\hline & Pep Ritger-pas Equation & $\ln Q=0.34 \ln t+3.75$ & 0.961 \\
\hline \multirow[t]{5}{*}{ Lnxc-PLGA-MS-gels } & Zero-Order Kinetics & $Q=0.24 t+20.7$ & 0.922 \\
\hline & First-Order Kinetics & $\ln (100-Q)=-0.0050 t+4.4$ & 0.976 \\
\hline & Higuchi Equation & $\mathrm{Q}=4.56 \mathrm{t}^{1 / 2}+8.83$ & 0.987 \\
\hline & Weibull Equation & $\ln \ln (I /(I-Q / / 00)=0.51 \ln t+2.23$ & 0.995 \\
\hline & Pep Ritger-pas Equation & $\ln Q=0.40 \ln t+2.23$ & 0.988 \\
\hline
\end{tabular}

Table 3 The pharmacokinetic parameters of Lnxc in the articular

\begin{tabular}{lllll}
\hline Analytical method & Parameters & Lnxc-MS-PLGA-gels & Lnxc-PLGA-MS & Lnxc-sol \\
\hline Two Chamber Model & $\mathrm{t}_{1 / 2 \alpha}(\mathrm{h})$ & 3.04 & 5.01 & 0.53 \\
& $\mathrm{t}_{1 / 2 \beta}(\mathrm{h})$ & 67.58 & 64.37 & 2.33 \\
& $\mathrm{~V}_{1}(\mathrm{~L} / \mathrm{kg})$ & 0.17 & 0.21 & 0.97 \\
& $\mathrm{CL}(\mathrm{L} / \mathrm{h} / \mathrm{kg})$ & 0.004 & 0.005 & 0.45
\end{tabular}

10, Lnxc-Sol groups were decreased quickly and drug concentration cannot be found until at $8 \mathrm{~h}$ whether in articular tissues or synovial, however, the Lnxc-PLGA-MS groups and the Lnxc-PLGA-MS-gels groups were decreased slowly with date till $13.5 \mathrm{~d}$. After injection with Lnxc-PLGA-MS-gels at $0.5 \mathrm{~h}$, the concentration of Lnxc in articular cavity was lower than Lnxc-PLGA-MS which was due to the reduction of the burst release. The drug released from the Lnxc-PLGA-MS and the Lnxc-PLGA-MS-gels was absorbed into the blood quickly, so the Lnxc-PLGA-MS-gels has a better sustained effect by contrast, meanwhile, gels could reduce the initial burst at the start of intra-articular injection.

The pharmacokinetic parameters are summarized in Table 3. The area under the curve (AUC) of Lnxc-PLGA-MS-gels group is 1.29 times more than the Lnxc-PLGA-MS group, the drug mean retention time (MRT0- $\infty$ ) of Lnxc-PLGA-MS-gels group and Lnxc-PLGA-MS group is 34.72 times and 33.31 times than the Lnxc solution group, respectively. That all indicated that Lnxc-PLGA-MS-gels group and Lnxc-PLGA-MS group has less drugs leaked to the systemic cycle of rats from the articular cavity, and then Lnxc-PLGA-MS-gels group could reduce the sudden burst and maintained a relatively high level in targeted site. Many attempts have been made to reduce the initial burst drug release associated with temperature-sensitive gels systems due to slow in vivo sol-gel transition. ${ }^{24}$ According to the data analysis in vitro and in vivo, the chitosan temperature-sensitive gels has prepared not only reduced the burst release of Lnxc-PLGA-MS but prolonged retention time of Lnxc in the joint cavity, which could improve the efficiency of Lnxc and decrease the side effects. 


\begin{tabular}{|c|c|c|c|c|}
\hline Analytical method & Parameters & Lnxc-MS-PLGA-gels & Lnxc-PLGA-MS & Lnxc-sol \\
\hline & 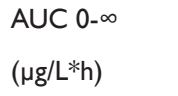 & 974708.41 & 754274.16 & 8922.31 \\
\hline \multirow[t]{2}{*}{ Statistical Moment } & MRT $0-\infty(h)$ & 83.67 & 80.27 & 2.41 \\
\hline & $\mathrm{C}_{\max }(\mu g / \mathrm{L})$ & 19947.69 & 17904.76 & 2627.7 \\
\hline
\end{tabular}

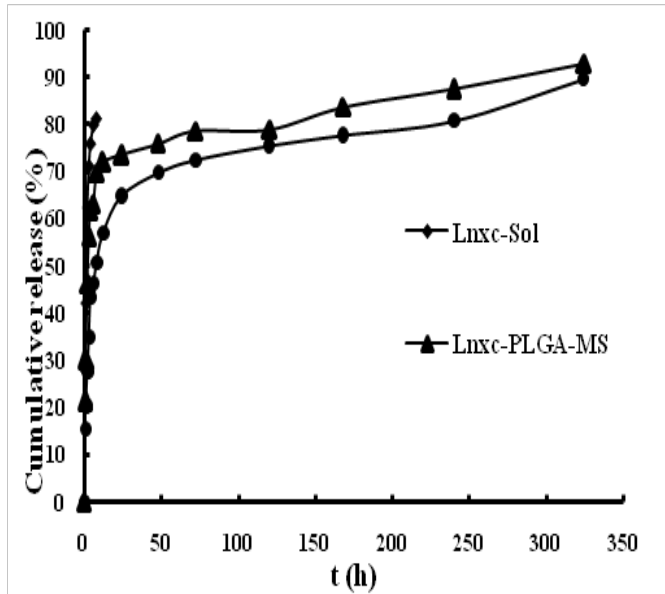

Figure 7 In vitro release of Lnxc, Lnxc-PLGA-MS and Lnxc-PLGA-MS-gels.

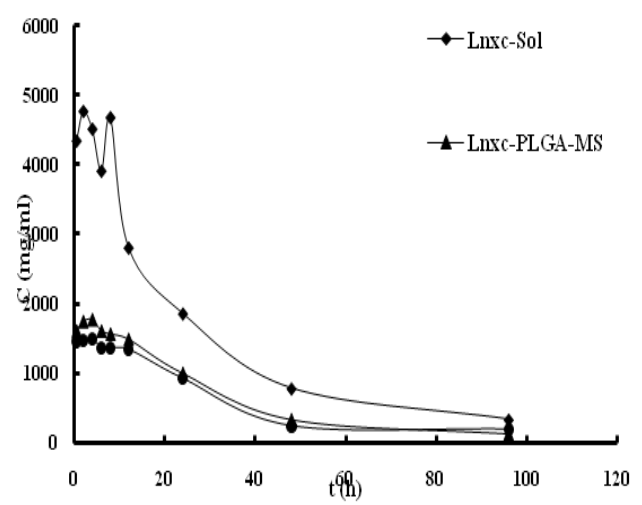

Figure 8 Mean plasma concentration-time curves of Lnxc.

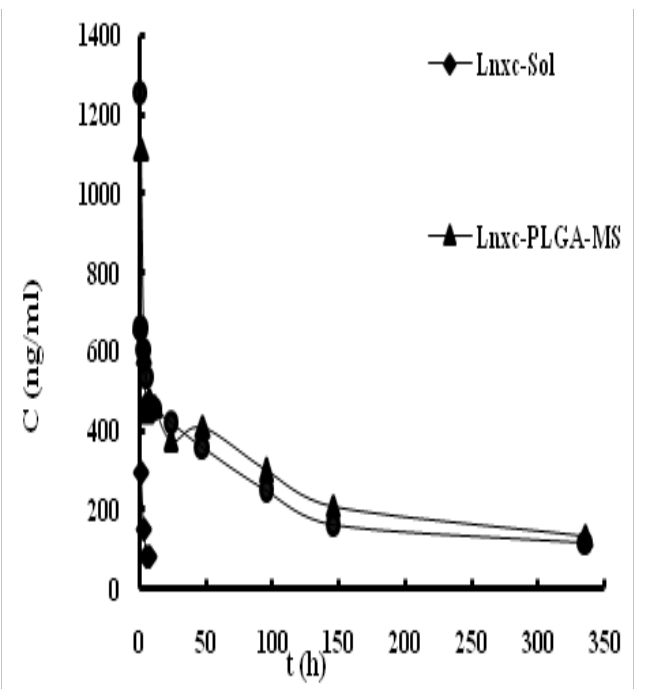

Figure 9 Mean residual concentration-time curves of Lnxc in the articular tissues.

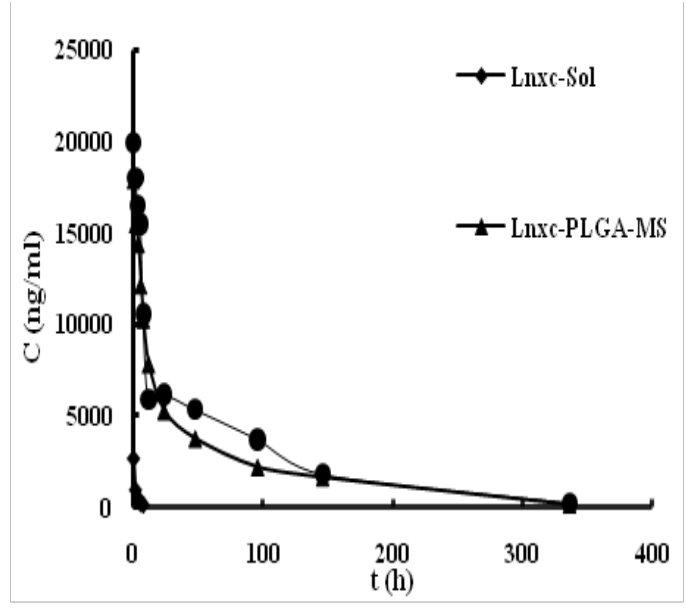

Figure 10 Mean residual concentration-time curves of Lnxc in the articular synovial

\section{Conclusion and outlook}

In this study, an intra-articular drug delivery system was prepared based on $\mathrm{CS} / \beta$-GP-temperature-sensitive gels that can spontaneously form a "drug depot" in the articular after injection and increase the retention time of drugs. ${ }^{18,25}$ Hydrogels are macromolecular networks, which can absorb large amounts of water while maintaining a distinct three-dimensional structure. Hydrogels of chitosan are currently receiving much attention for medical and pharmaceutical applications, as chitosan is biocompatible, biodegradable, non-toxic and easily available. ${ }^{26,27}$ Loaded with Lnxc-PLGA-MS, the combined gels showed increased viscosity and reinforced intensity, and had sustained drug releasing for $13.5 \mathrm{~d}$ in vitro studies. The results of determination of targeting efficiency revealed that the gels containing microspheres delivery system seemed to effectively reduce the leakage to the systematic circulation and had superior retention effect than microspheres. The present study demonstrate that intra-articular administration of temperature-sensitive gels reduce the release burst effect of Lnxc-PLGA-MS and enhance drug targeting in joint cavity due to the prolonged stagnation time of drug in the joint, meanwhile, this drug sustained-releasing system reduced systemic adverse reactions and had superior anti-inflammation effects than drug solution, achieving a novel drug delivery system. As for the treatment strategies by this combination system, there are still needs for further long-term studies.

\section{Acknowledgments}

The authors would like to express thanks to the School of Pharmaceutical Science, Shandong University for providing the required infrastructure to carry out the study.

\section{Declaration of interest}

The work described has not been submitted elsewhere for publication in whole or in part, and all the authors listed have approved 
the manuscript that is enclosed. The authors report no conflicts of interest. The authors alone are responsible for the content and writing of this article.

\section{References}

1. Skjodt NM, Davies NM. Clinical pharmacokinetics of lornoxicam. A short half-life oxicam. Clin Pharmacokinet. 1998;34(6):421-428.

2. Rivera F, Bertignone L, Grandi G, et al. Effectiveness of intra-articular injections of sodium hyaluronate-chondroitin sulfate in knee osteoarthritis:a multicenter prospective study. J Orthop Traumatol. 2016;17(1):27-33.

3. Abdelbary GA, Aburahma MH. Oro-dental mucoadhesive proniosomal gel formulation loaded with lornoxicam for management of dental pain. J Liposome Res. 2015;25(2):107-121.

4. Zandstra J, van Beuge MM, Zuidema J, et al. Microsphere-Based Rapamycin Delivery, Systemic Versus Local Administration in a Rat Model of Renal Ischemia/Reperfusion Injury. Pharm Res. 2015;32(10):32383247.

5. Uematsu T, Sakai A, Ito $\mathrm{H}$, et al. Intra-articular administration of tachykinin $\mathrm{NK}_{1}$ receptor antagonists reduces hyperalgesia and cartilage destruction in the inflammatory joint in rats with adjuvant-induced arthritis. European Journal of Pharmacology. 2011;668(1-2):163-168.

6. Saricaoglu F, Dal D, Atilla P, et al. Effect of intra articular injection of lornoxicam on the articular cartilage \& synovium in rat. Indian $\mathrm{J}$ Med Res. 2008;127(4):362-365.

7. Schroeder S, Heuser A, Tellmann A, et al. Local tolerance of intra articular administration of lornoxicam into the rabbit knee joint. Rheumatol Int. 2012;32(9):2661-2667.

8. Ramazani F, Chen W, van Nostrum CF, et al. Strategies for encapsulation of small hydrophilic and amphiphilic drugs in PLGA microspheres: State-of-the-art and challenges. Int J Pharm. 2016;499(1-2):358-367.

9. Casalini T, Rossi F, Lazzari S, et al. Mathematical modeling of PLGA microparticles: from polymer degradation to drug release. Mol Pharm. 2014;11(11):4036-4048.

10. Goto N, Okazaki K, Akasaki Y, et al. A single intra-articular injection of fluvastatin-PLGA microspheres reduces cartilage degradation in rabbits with experimental osteoarthritis. J Orthop Res. 2017;35(11):2465-2475.

11. Cilurzo F, Selmin F, Minghetti P, et al. Design of methylprednisolone biodegradable microspheres intended for Intra-articular administration. AAPS Pharm Sci Tech. 2008;9(4):1136-1142.

12. Eswaramoorthy $\mathrm{R}$, Chang $\mathrm{CC}$, Wu SC, et al. Sustained release of PTH(1-34) from PLGA microspheres suppresses osteoarthritis progression in rats. Acta Biomater. 2012;8(6):2254-2262.

13. Ko JY, Choi YJ, Jeong GJ, et al. Sulforaphane-PLGA microspheres for the intra-articular treatment of osteoarthritis. Biomaterials. 2013;34(21):5359-5368.
14. Abd-Allah H, Kamel AO, Sammour OA. Injectable long acting chitosan/tripolyphosphate microspheres for the intra-articular delivery of lornoxicam: Optimization and in vivo, evaluation. Carbohydrate Polymers. 2016;149:263-273.

15. Wang J, Wang BM, Schwendeman SP. Characterization of the initial burst release of a model peptide from poly(D,L-lactide-co-glycolide) microspheres. J Control Release. 2002;82(2-3):289-307.

16. Ruel-Gariépy E, Chenite A, Chaput C, et al. Characterization of thermosensitive chitosan gels for the sustained delivery of drugs. Int $J$ Pharm. 2002;203(1-2):89-98.

17. Ruel-Gariépy E, Shive M, Bichara A, et al. A thermosensitive chitosan-based hydrogel for the local delivery of paclitaxel. Eur J Pharm Biopharm. 2004;57(1):53-63.

18. Qi X, Qin X, Yang R, et al. Intra-articular administration of chitosan thermosensitive In Situ hydrogels combined with diclofenac sodiumloaded alginate microspheres. J Pharm Sci. 2016;105(1):122-130.

19. Zhang Z, Bi X, Li H, et al. Enhanced targeting efficiency of PLGA microspheres loaded with Lornoxicam for intra-articular administration. Drug Deliv. 2011;18(7):536-544.

20. Zhang Z, Huang G. Intra-articular lornoxicam loaded PLGA microspheres: enhanced therapeutic efficiency and decreased systemic toxicity in the treatment of osteoarthritis. Drug Deliv. 2012;19(5):255-263.

21. Tian H, Huang G, Zhu S, et al. The preparation and in vitro evaluation of Lornoxicam loaded chitosan-temperature-sensitive gels for intra-articular administration. Shandong Medical Journal. 2017;10:100-102.

22. Santos SS, Lorenzoni A, Ferreira LM, et al. Clotrimazole-loaded Eudragit $^{\circledR}$ RS100 nanocapsules: preparation, characterization and in vitro evaluation of antifungal activityagainst Candida species. Mater Sci Eng C Mater Biol Appl. 2013;33(3):1389-1394.

23. Venkateswarlu V, Manjunath K. Preparation,characterization and in vitro release kinetics of clozapine solid lipid nanoparticles. J Control Release. 2004;95(3):627-638.

24. Priya James H, John R, Alex A, et al. Smart polymers for the controlled delivery of drugs-a concise overview. Acta Pharm Sin B. 2014;4(2):120-127.

25. Kim T, Seol DR, Hahm SC, et al. Analgesic effect of intra-articular injection of temperature-responsive hydrogel containing bupivacaine on osteoarthritic pain in rats. BioMed Research International. 2015;2015(2015):9.

26. Khurma JR, Nand AV. Temperature and $\mathrm{pH}$ sensitive hydrogels composed of chitosan and poly(ethylene glycol). Polymer Bulletin. 2008;59(6):805-812.

27. Zhu AM, Hua Chen J, Liu QL, et al. Controlled release of berberine hydrochloride from alginate microspheres embedded within carboxymethyl chitosan hydrogels. Journal of Applied Polymer Science. 2015;120(4):2374-2380. 